\title{
FORMACIÓN DE LAS MUJERES, EMPODERAMIENTO E INNOVACIÓN RURAL ${ }^{1}$
}

\author{
Ana María Porto Castro \\ Departamento de Métodos de Investigación y Diagnóstico en Educación. Universidade de Santiago de Compostela \\ anamaria.porto@usc.es \\ Montserrat Villarino Pérez \\ Departamento de Geografía. Universidade de Santiago de Compostela \\ montserrat.villarino@usc.es \\ Mireia Baylina Ferré \\ Departamento de Geografía. Universitat Autònoma de Barcelona \\ mireia.baylina@uab.es \\ Maria Dolors García Ramón \\ Departamento de Geografía. Universitat Autònoma de Barcelona \\ MariaDolors.Garcia.Ramon@uab.es \\ Isabel Salamaña Serra \\ Departamento de Geografía. Universitat de Girona \\ isabel.salamana@udg.edu
}

\section{RESUMEN}

En el marco de un espacio rural, mujeres formadas inician proyectos profesionales. Su iniciativa es importante no solamente por las prácticas que desarrollan y por sus efectos en el territorio sino también porque consiguen la permanencia de un colectivo social especialmente amenazado desde hace décadas. Los proyectos desarrollados muestran ideas, conocimientos, información, estrategia, alta capacidad de organización y de trabajo y red de relaciones. Para ello, la formación recibida es un recurso fundamental. Sus conocimientos las empoderan como mujeres profesionales y les permite superar barreras de género y de clase. Este artículo ahonda en el papel de la formación en la vida de las mujeres profesionales del medio rural de Galicia y Cataluña y en cómo determina la revitalización de estas áreas.

Palabras clave: género, formación, innovación, medio rural.

Fecha de recepción: mayo 2013.

Fecha de aceptación: febrero 2014.

1 Este artículo muestra parte de los resultados del proyecto que lleva por título MUJERES, TRABAJO Y RURALIDAD: ESTRATEGIAS INNOVADORAS PARA EL DESARROLLO DE UN PROYECTO PROFESIONAL Y PERSONAL (2011-0004-INV-00024), financiado por el Instituto de la Mujer, Ministerio de Sanidad, Política Social e Igualdad. 


\section{ABSTRACT}

In the context of a rural space, educated women initiate innovative projects. Their initiative is important not only for the practices they develop and for the effects they generate in the area but also because they achieve the permanence of a particular social group that has been threatened since decades. Their projects show ideas, knowledge, information, strategy, organizational skills, high work capacity and social networks. In their ability to initiate professional projects, education and training become crucial resources. Their knowledge empowers them as professional women and enables them to overcome barriers of gender and class. This article tries to delve into the role of education in the lives of professional rural women in Galicia and Catalonia and to examine how education may determine the revitalization of these areas.

Keywords: gender, education, innovation, rural space.

\section{INTRODUCCIÓN}

Desde hace algunas décadas el medio rural en España ha experimentado cambios muy notables en relación a la base económica y la ocupación, al incremento de las segundas residencias, al retorno de antiguos emigrantes y a la llegada de inmigrantes extranjeros, en ritmo e intensidad distintos según los contextos geográficos (García, 2011). Estos cambios han venido marcados por los procesos globales de producción y consumo que afectan a todos los territorios sin excepción. Según Woods (2011), la globalización contemporánea se distingue de otras etapas globalizadoras porque abarca todos los rincones del planeta, es capaz de establecer conexiones inmediatas (sociales, financieras...) y porque se desarrolla bajo los fundamentos de la ideología neoliberal. La expansión de un mercado libre global y los principios neoliberales han producido un discurso del medio rural global como un espacio económico singular (2011: 157). Ante esta realidad, los espacios rurales, de acuerdo a las directrices de la Unión Europea orientadas a promover el desarrollo endógeno de estos territorios (van der Ploeg et al., 2000), se han movilizado para dinamizar sus economías a partir de los recursos locales sabiendo que hay un interés desde la ciudad por lo rural y también reconociendo una necesidad propia (la emigración a la ciudad ya no es una solución ante la escasez de trabajo, la precariedad salarial o el elevado coste de la vivienda) (García, 2011).

Muchas áreas rurales comparten las formas de bienestar de la mayoría de regiones urbanas gracias a la diversificación de actividades y a una economía manifiestamente interconectada (Aldomà, 2009) y, en general, existe un imaginario más positivo de la vida rural, incluso idealizado. Esta atracción hacia las áreas rurales conlleva la apertura de nuevos nichos de trabajo tales como el turismo rural, y han reformulado la estructura socioeconómica del territorio (Prados, 2000; Hoggart y Paniagua, 2002; Bock, 2004; Cànoves et al., 2004; Morris y Little, 2005; García Sanz, 2004; Baylina y Salamaña, 2006; Woods, 2011). Sin embargo, como señala Woods (2011), no todas las localidades rurales están igualmente equipadas para competir en la economía global (factores estructurales) ni la respuesta local (iniciativa individual y colectiva) tiene porque ser la misma. En distintos lugares se han producido nuevas y distintas acciones en función de las capacidades y recursos de los actores locales. Y en 
este sentido, las mujeres han constituido un capital social y humano muy importante para el desarrollo rural y local en el medio rural a nivel económico, social y político (Garcia Ramon y Baylina, 2000; Prados, 2000; Pallarès et al., 2004; Nieto, 2004; Tulla et al., 2012; Casellas et al., 2013).

En este contexto, en España el número de mujeres en el medio rural sigue siendo inferior al de hombres, y sobre todo en edades intermedias (tasa de masculinidad entre la población de 20 a 64 años es de 103,9), consecuencia todavía de la huida ilustrada que ha empujado a las mujeres jóvenes a estudiar y alejarse de una comunidad fuertemente patriarcal (Whatmore, 1990; Bordieu, 2004; Camarero et al., 2009, Ministerio de Medio Ambiente, y Medio Rural y Marino, 2011); trabajan mayoritariamente en el sector de servicios (comercio, educación, sanidad: el 78\% de las mujeres entre los 20 y los 64 años frente al $41 \%$ de los hombres de la misma franja de edad) y superan a los hombres en la inactividad y en el desempleo; tal como se recoge en el Diagnóstico de la Igualdad de Género en el Medio Rural (2011), entre la población rural comprendida entre los 20 y 64 años, la tasa de ocupación masculina es del $72 \%$ frente al $49 \%$ de la femenina, y la de inactividad es del $15 \%$ y $38 \%$ de hombres y mujeres, respectivamente, con un dato de gran interés: de la población inactiva, las 3/4 partes de los hombres lo son como pensionistas, mientras que esa misma proporción entre las mujeres se debe a su ocupación en el trabajo doméstico y de cuidados. Sin embargo, aumenta muy significativamente su nivel de formación, que es superior al de los hombres (el 22\% de las mujeres entre los 20 y 64 años tienen estudios superiores frente al $15 \%$ de los hombres de la misma franja de edad), y aumenta también el número de profesionales autónomas, por lo menos en el sector agrario (59\% mujeres vs 54\% hombres) (García, 2011). Y en esta nueva realidad, algunas mujeres formadas deciden quedarse o instalarse en el medio rural para emprender su reto profesional, innovador en su contexto y comunidad. Sus motivaciones para residir en lo rural y su construcción de arraigo son diversas (Villarino et al., 2013; Carbó et al., 2014). Además de factores tangibles como el aumento de la demanda ocupacional en los servicios, y, sobre todo, la posibilidad de desplazarse a otros puntos para trabajar o el trabajo a distancia, un mayor aprecio de la sociedad hacia lo rural y la mejora de sus condiciones de vida han hecho disminuir las reticencias de las generaciones más jóvenes a permanecer en el lugar (Villarino et al., 2013). Como señala Díaz $(2005,2006)$, tras veinte años de cambios efectivos en las áreas rurales, el sentido de apego y pertenencia ha aumentado.

Ahora bien, los elementos materiales son tan cruciales como la creación de una nueva identidad de género, construida a partir de un proceso de redefinición de lo rural (Sampedro, 2009). El arraigo al lugar actual se constituye en base a una nueva identidad rural -en la que la movilidad y el estatus laboral son esenciales- y es comparable a aquellos procesos que podrían darse en la ciudad. Para ello, el capital cultural y social resulta fundamental, convirtiéndose en el recurso esencial sobre el que basar iniciativas vitales y laborales.

Las iniciativas innovadoras con frecuencia implican el establecimiento de nuevas empresas o el trabajo como profesionales freelance, ambas actividades asociadas tradicionalmente al mundo masculino pese al notorio incremento de negocios en manos de mujeres detectado en todo el mundo en las últimas décadas (Hanson, 2009). Dado que el empresariado se contempla como masculino la presencia de mujeres denota ya un cambio en la construcción del género. Las mujeres emprendedoras están extendiendo los significados de género sobre el empresariado, desestabilizan su asociación con la masculinidad y potencialmente crean 
nuevas subjetividades para sí mismas y nuevas oportunidades para que otras mujeres puedan ganarse la vida. También amplían las concepciones de género en sus comunidades y más allá de ellas. Por un lado, el emprendimiento se entiende aquí como un proceso local que tiene la capacidad de transformar tanto un lugar como al agente emprendedor, convirtiéndolo en la persona que posee un negocio, asume los riesgos asociados, se enfrenta con las incertidumbres de coordinar unos recursos y está al cargo de la gestión diaria del negocio (Hanson, 2009). Y, por otro lado, la innovación se entiende como la acción de mudar o alterar algo, introduciendo novedades; o la creación de un producto y su introducción en el mercado (DRAE, 2001).

Este trabajo pretende ahondar en el papel de la formación en las iniciativas laborales de mujeres profesionales del medio rural de Cataluña y Galicia. Se parte de la idea que en su capacidad para iniciar su actividad laboral la formación recibida es un recurso fundamental que las puede empoderar como profesionales y permitir superar barreras de género y de clase. Al mismo tiempo, se considera que en los itinerarios formativos, las decisiones y acontecimientos que se suceden pueden ser explicados desde unas relaciones de género determinadas en la unidad familiar y en la comunidad rural.

A continuación se expone el perfil de las mujeres consideradas, la metodología utilizada y algunos datos básicos sobre las áreas de estudio; en el tercer apartado se abordan los estereotipos y las expectativas formativas de las mujeres y de la familia; y en el cuarto apartado se analiza el papel de la formación en el itinerario personal y profesional innovador de las mujeres. Finalmente, se apuntan algunas conclusiones.

\section{METODOLOGÍA Y ÁREAS DE ESTUDIO}

Esta investigación se basa en la experiencia de 60 mujeres de Cataluña y de Galicia que decidieron desarrollar su proyecto de vida y su proyecto profesional en el medio rural. La mayoría $(66,6 \%)$ nacieron en el medio rural pero generalmente vivieron en áreas urbanas en alguna etapa de su vida; algunas $(8,4 \%)$ nacieron en áreas urbanas pero tenían vínculos familiares con lo rural; y una cuarta parte $(25 \%)$ proceden de zonas urbanas. El perfil corresponde a una mujer entre 30 y 60 años de edad, con estudios universitarios $(74,6 \%)$, casada o con pareja heterosexual $(83,3 \%)$ y que mayoritariamente tiene hijos $(72,2 \%)$. Algo más de la mitad de estas mujeres son gerentes de su propia empresa o autónomas $(52,6 \%)$ y la mayoría trabajan en el sector servicios $(72,9 \%)$ (turismo, educación, traducción, atención a mayores, entre otros) seguido del sector agrario $(20,4 \%)$ (producción y transformación de productos ecológicos, forestal, producción con denominación de origen, etc.). Hemos utilizado una metodología cualitativa a través de las entrevistas en profundidad (30 en cada región, de dos horas de duración cada una, realizadas en el domicilio de la entrevistada) que han sido grabadas, transcritas y codificadas con el programa Atlas ti, y observaciones participantes realizadas por cada entrevistadora. Además se han hecho 20 entrevistas semiestructuradas (10 en cada región) a informantes cualificados/as para obtener información de calidad y de primera mano sobre el desarrollo rural y cuestiones de género a fin de contextualizar las experiencias y las percepciones de las mujeres entrevistadas. Estas entrevistas también fueron grabadas, transcritas y codificadas con el citado programa informático. 
Cataluña y Galicia son dos regiones españolas contrastadas. Cataluña (7,5 millones de habitantes, INE) fue motor económico en España por su industria, mientras que Galicia (2,7 millones de habitantes, INE) tuvo una economía de base tradicional y con un fuerte proceso emigratorio. Actualmente, en ambas regiones la estructura económica se apoya en el sector servicios aunque en Galicia el empleo en el sector primario tiene una cierta relevancia (7\% de ocupados, INE). También la población rural en ambas áreas es diferente (31\% en Galicia y 19\% en Cataluña), y entre 1996 y 2009 ésta experimentó un decrecimiento del -7,3\% en Galicia y un aumento del 11,8\% en Cataluña (García, 2011); esta evolución se debe en el contexto gallego al envejecimiento de la población rural, mientras que el aumento en el catalán responde a un proceso de reruralización tanto por población local como por inmigrantes, aunque esto no signifique un aumento en la ocupación agraria (1\% de ocupados en Cataluña, INE).

\section{EXPECTATIVAS FORMATIVAS}

\section{III.1. Estereotipos de género}

Los factores psicológicos y los factores sociales, entre ellos la socialización diferencial de género femenino y masculino, los estereotipos sobre características y roles de hombres y mujeres dominantes en una cultura o las normas culturales (Elejabeitia y López-Sáez, 2003:177) son fundamentales para explicar la trayectoria personal y profesional de las personas. Las creencias consensuadas sobre las diferentes características de mujeres y hombres en nuestra sociedad (Mosteiro, 2010: 240) llevan implícitas la existencia de una dicotomía de rasgos de personalidad, roles, características físicas y ocupaciones que se asocian tradicionalmente a hombres y mujeres (Deaux y Lewis, 1983), y tienen una gran influencia en el individuo, en su percepción del mundo y de sí mismo y en su conducta. Niños y niñas empiezan a adquirir los estereotipos de género casi al mismo tiempo que toman conciencia de su identidad de género y hacia los ocho años ya tienen la capacidad para emitir juicios estereotipados de mujeres y hombres (Martin, Wood y Little, 1990).

Desde una perspectiva sociocultural, los estereotipos de género surgen del medio social y reflejan claramente la cultura. Eagly y Steffen (1984) y Williams y Best (1990) defienden que el contexto social es el que ejerce una mayor influencia sobre la estereotipia de género, ya que el desarrollo precoz de los estereotipos de género solo puede explicarse teniendo en cuenta el trato diferencial que niñas y niños reciben desde el momento en que llegan al mundo y que determina un comportamiento y unas expectativas radicalmente diferentes respecto a ellas y ellos en función de su sexo biológico. En la reproducción de los estereotipos de género el papel de la familia y la escuela, principales agentes de socialización, es fundamental pues se convierten en referentes del comportamiento adecuado para cada sexo en la sociedad.

En el proceso de socialización primaria, el contexto familiar y la actuación de los progenitores inciden directamente en la aparición y consolidación de los estereotipos de género. El modo diferenciado de vestir a los bebés, adjudicando colores apropiados para cada sexo, el encauzamiento hacia intereses y aficiones adecuadas a su rol, mediante la compra de juguetes o el fomento de motivaciones específicas, son algunos de los ejemplos 
de la socialización diferencial que los padres y otros agentes socializadores llevan a cabo con los niños/as, condicionando de este modo sus actitudes y comportamientos en la vida adulta (Mosteiro, 2010: 244).

En la etapa escolar el comportamiento de los progenitores hacia sus hijos e hijas es diferencial en sus expectativas, referidas sobre todo al desarrollo de determinadas capacidades relacionadas directa o indirectamente con los aprendizajes escolares. Las madres y los padres consideran que existen importantes diferencias entre chicos y chicas en capacidad verbal, viso-espacial y matemática (Shaffer, 2000). Estas diferencias responden a ciertas formas de comportamiento aprendidas, cuyo origen se sitúa en los distintos modos de interacción que los progenitores establecen con su descendencia dependiendo del sexo biológico de ésta (Barberá, 2005).

A lo largo de la adolescencia, etapa en la que se produce una redefinición y búsqueda de la identidad sexual y de género, las actitudes, valores y normas dominantes en nuestra sociedad acerca de la masculinidad y feminidad influirán decisivamente en los chicos y chicas, que siguen las pautas de comportamiento asociadas a los roles de género convencionales influenciados por las actuaciones de los progenitores y de los grupos de iguales. Madres y padres adoptan un rol de proteccionismo respecto a sus hijas tratando de ejercer un mayor control en lo que se refiere a las salidas, horarios y amistades que con sus hijos, a quienes se les concede una mayor libertad (Valentine y McKendrick, 1997; Valentine, 2004; Hopkins, 2010).

En definitiva, «El sistema educativo familiar es, junto con el escolar, uno de los mecanismos de socialización de mayor influencia sobre el comportamiento personal y social de los individuos. Desde la más tierna infancia se nos inculcan una serie de valores y una serie de expectativas que van a prevalecer a lo largo de nuestra vida -de una manera más o menos consciente- y van a condicionar las decisiones que tomemos, así como las elecciones que hagamos» (Sainz, López-Sáez y Lisbona, 2004: p. 112).

Así pues, los estereotipos y roles tradicionales de género determinan la percepción de las elecciones que realizamos. «De esta forma, las mujeres se concentran en profesiones vinculadas con el estereotipo femenino, es decir, profesiones de atención y cuidado de personas, y los hombres en profesiones de carácter técnico o científico» (Candela, Barberá y Ramos, 2009: p. 29).

De forma bastante general, las mujeres entrevistadas -independientemente del lugar, posición social o económica- plasman los estereotipos femeninos cuando contestan a la pregunta ‘¿qué querían ser de mayores?'. En sus elecciones se decantan por profesiones muy feminizadas, asociadas al cuidado y al bienestar de las otras personas, que coinciden con la representación social de los roles femeninos (Elejabeitia y López Sáez, 2003) y, en general, con el proceso de construcción histórica de la feminidad (Nash, 1995). Profesiones como profesora, maestra, peluquera, pediatra, enfermera, veterinaria o modista, aparecen entre sus preferencias de jóvenes:

«Pediatra y bailarina». (Carmen, 50, psicóloga/psicóloga, Cataluña).

«Peluquera». (Rosalba, 49, trabajo social/profesora, Cataluña).

«Desde profesora, a veterinaria y bailarina...». (Carlota, 45, farmacia/agricultora, Galicia).

«...quise ser maestra para personas con problemas». (Judit, 47, pedagogía/directora escuela infantil, Galicia). 
«Creo que ser abogada... Quería ayudar a los demás». (Claudia, 39, publicidad y periodismo; graduado social/propietaria librería, Galicia).

«Enfermera». (Elisabet, 51, traducción e interpretación/gerente de Albergue, Cataluña).

«Me gustaba aspirar a ser médico o alguna cosa así; quería ayudar a alguien». (Candela, 62, administrativo/administrativa, Galicia).

\section{III.2. La construcción de las expectativas}

Las expectativas, entendidas como la probabilidad subjetiva de que determinadas acciones producirán ciertos resultados (Salanova et al., 1996), son procesos psicológicos que afectan a la motivación y desempeñan un papel principal en la elección profesional. En este sentido, las expectativas de éxito, la confianza en las propias habilidades para lograr el éxito y la percepción de competencia son reconocidas como variables importantes en el proceso de toma de decisiones (Hollinger, 1983; Betz y Hackett, 1986), y en este proceso, los hombres y las mujeres tienen expectativas distintas y en áreas de conocimiento diferentes (Eccles, 1994; Hyde et al., 1990; Swim, 1994). En un estudio llevado a cabo por Jozefowicz, Barber y Eccles (1993) se comprobó que las chicas tenían menores expectativas de éxito que los chicos en las profesiones del área científica y en las profesiones consideradas masculinas. Por otra parte, los chicos tenían menores expectativas de éxito que ellas en las profesiones típicamente femeninas, algo que refleja los tradicionales roles de género estereotipados.

En este sentido Hernández Franco (2001) subraya que hay que considerar que el género influye de modo significativo en las expectativas de autoeficacia y a través de éstas en la autoestima vocacional y en los intereses profesionales. Las chicas se consideran menos eficaces que los chicos en aquellas actividades intelectuales que a través de los estereotipos sociales se relacionan con los hombres.

Es también importante considerar que los progenitores y docentes tienen distintas expectativas de logro y varían sus explicaciones del éxito y del fracaso en función del sexo del descendiente o discente respectivamente.

«Porque mi padre, en alguna ocasión, lo tenían medio convencido: «A la niña, la podrías enviar a costura no sé dónde...»; «Al niño lo podrías enviar a aprender de relojero no sé con quién...». (Jana, 59, filología gallega/profesora de secundaria, Galicia).

«A las mujeres nos tenía un poco relegadas... A mi hermano le dio la oportunidad de salir al extranjero; ha estado en EE.UU. estudiando, ha estado a costes pagados en Inglaterra... (...) A nosotras esas oportunidades no nos las dio nunca. La idea de nuestro padre ha sido la de siempre: la mujer se queda en casa, cuida de los niños y cuida de la casa...». (Gema, 37, administrativa/empresaria, Galicia).

«Mi padre me decía: lástima que no seas un chico. Porque veía en mí a una persona emprendedora». (Coloma, 61, secretariado dirección/gerente centro de educación especial, Cataluña). 
«Primero hice Formación Profesional; lo hice conducida por las maestras de Educación Básica. [Según ellas] enfrentarse a un BUP era imposible y nos dijeron que nos decantásemos por la Formación Professional. A mí no me gustaba nada pero me vi obligada a hacer lo que me decían». (Rosalba, 49, trabajo social/ profesora instituto, Cataluña).

En efecto, las expectativas de los padres y las madres condicionan en gran medida las expectativas y el concepto que tienen las mujeres de sí mismas. Y en los deseos de los progenitores también influyen la situación socioeconómica familiar y el entorno social y cultural en el que se mueven.

En la mayoría de los casos, se constata que padres y madres apoyan y animan a sus hijos e hijas a adquirir formación y cuanta más alta, mejor. La razón principal es el ascenso social, de tal modo que es muy frecuente que los progenitores quieran que sus hijos/as lleguen más lejos que ellos, sobre todo en las familias con menor nivel sociocultural, y que cualquier esfuerzo económico se da por bueno ya que los estudios son la mejor herencia que pueden dejarles.

«Ellos querían que estudiase, cuanto más mejor. Nunca me dijeron haz esto o haz aquello. Ellos solo querían que yo estudiase y los superase; si ellos no habían podido estudiar, querían que lo hiciese yo». (Thais, 56, psicología/propietaria librería, Cataluña).

«Yo empecé mis estudios en una escuela pública, pero allí hubo problemas y no dudaron un momento en sacarnos a los cinco hijos y llevarnos a una escuela privada. La mejor herencia que me han dejado mis padres han sido mis estudios». (Diana, 43, doctora en ingeniera agrícola; máster en ciencias políticas/investigadora en alimentos, Cataluña).

«Siempre quiso que sus hijos estudiasen y se preparasen. Aunque él no tenía estudios, siempre dijo que quería que estudiáramos. Decía que no nos iba a dejar dinero, pero que nos iba a permitir prepararnos». (Carmina, 61, maestra/responsable la oficina agraria comarcal, Galicia).

«Soy la primera de mi familia que estudia y acaba una carrera; [...]. Yo tenía que estudiar. Mi madre pretendía que fuera juez porque siempre piensa que desde la justicia se hacen muchas cosas». (Carla, 41, historia; máster en archivística; máster en museología y gestión de patrimonio/subdelegada patrimonio del obispado, Cataluña).

En ocasiones, un nivel socioeconómico bajo también puede mermar las expectativas de los padres y de las propias hijas que saben que solo pueden aspirar a cursar la enseñanza obligatoria para ponerse a trabajar a continuación.

«La familia, económicamente, siempre fue muy ajustada y recuerdo que mi padre siempre nos decía «O estudiáis con beca o aquí no se puede estudiar». Mi padre 
sabía que no nos podía pagar unos estudios... (...). Lo que sí que recuerdo es que ese tipo de comentarios lo que hacían era frenar un poco nuestras expectativas...». (Paz, 41, bachillerato/administrativa empresa privada, Galicia).

«Cuando salimos del colegio, muchas compañeras fueron a estudiar a Lugo o a una academia de costura; yo no pude ir porque mi madre enfermó y tuve que quedarme en casa para ayudar con los trabajos de la agricultura. Nunca pude aprender nada, pero como me gustaba tanto, intentaba aprender por mí misma». (Jimena, 52, estudios primarios/agricultora, Galicia).

Y los trabajos a los que pueden acceder están muy condicionados por ser mujeres y por el lugar en el que viven; es el caso de las Rías Baixas en donde las mujeres de nivel socioeconómico bajo trabajaron tradicionalmente en la industria conservera.

«Nosotros somos 6 hermanos y, en mi casa, apoyaban a los hombres. Yo quería estudiar. A mí, me dieron a elegir: o ir a coser o a la fábrica de Massó, donde trabajaba mi madre. A mí la fábrica de Massó no me gustaba; cuando pasaba por delante me aguantaba la respiración... Pensaba que no iba a resistir ese mal olor... Como me dieron a elegir y yo a la fábrica no quería ir, pues fui a aprender a coser». (Verónica, 54, graduado escolar/presidenta de asociación cultural, Galicia).

En el caso de los progenitores que tienen estudios universitarios la formación superior para sus descendientes no se cuestiona, ni por parte de los padres ni tampoco por parte de hijos e hijas.

«Sí que invirtieron mucho en nuestra educación y fuimos a escuelas privadas, pero... Nos estimularon mucho cuando fuimos a la universidad, (...) Nos estimulaban mucho. Nuestros padres son universitarios, mi padre y mi madre». (Megan, 48, humanidades/profesora de inglés, Cataluña).

Ahora bien, los argumentos de las madres y los padres ofrecen matices distintos. Ellas instan a sus hijas a estudiar como medio para ser independientes, algo que no se observa en las expectativas manifestadas por los padres. Las mujeres ven la educación como un modo de aspirar a una vida diferente a la de agricultora, a salir del pueblo, en definitiva a no reproducir el modelo de las mujeres rurales de generaciones anteriores.

«Mi madre es muy luchadora y mucho de las que piensan que las mujeres se han de valer por sí mismas». (Thais, 56, psicología/propietaria librería, Cataluña).

«Mi madre siempre me dijo que fuera independiente, que hiciera lo que me diera la gana». (Carlota, 45, farmacia/agricultora, Galicia).

«Mi madre no decía demasiado...Lo que tenía muy claro es que quería que estudiásemos. Siempre había dicho que no se casaría con un pagès y se casó con un pagès...». (Abril, 36, turismo/patronato de turismo, Cataluña). 
«Siempre me apoyó para que hiciese algo, fuese carrera más grande o más pequeña. Nunca quiso que me quedara en el pueblo». (Delia, 45, administrativa/ administrativa empresa pública, Galicia).

Una idea que se reproduce también en las propias hijas:

«Yo, cuando era pequeña, lo que tenía claro era que no quería estar en el pueblo». (Abril, 36, turismo/patronato de turismo, Cataluña).

«También veía las grandes dificultades de otras mujeres de la época de mi madre que dependían de los maridos, y eso de la dependencia siempre fue una barrera para mí. Tenía claro que quería ser independiente. Entonces, veía claramente que quería tener estudios universitarios porque veía que era el modo de conseguir esta independencia». (Diana, 43, doctora en ingeniería agrícola; máster en ciencias políticas/investigadora en alimentos, Cataluña).

«Yo lo que quería era poder estudiar, salir y ser independiente. Quería poder vivir de mi trabajo». (Carmina, 61, maestra/responsable la oficina agraria comarcal, Galicia).

«Yo tenía en la cabeza viajar e irme fuera». (Alba, 38, comunicación audiovisual/ asesora de viajes, Cataluña).

En cambio, en las expectativas de los padres aparecen más los estereotipos femeninos sobre el tipo de profesión o sobre la capacidad potencial de las hijas:

«Cuando empecé biología, mi padre se disgustó un poco porque creía que era más fácil ser maestra o profesora». (Aina, 53, farmacia y biología/farmacéutica, Cataluña).

«Mi padre es mayor, tiene 92 años, y tiene una mentalidad antigua; no veía bien que las mujeres salieran de casa. De hecho, cuando yo me fui a Lugo se llevó un disgusto tremendo». (Jimena, 52, estudios primarios/agricultora, Galicia).

Y también se observa algún caso en que las mujeres manifiestan el papel secundario que tenían sus madres en la toma de decisiones:

«Bueno, ya sabes que antes quien llevaba la batuta era el cabeza de familia... (...). Antes las mujeres no tenían un duro. Era la forma de funcionar, no es que mi padre fuera malo... Él era el que gobernaba la propia casa». (Candela, 62, administrativo/administrativa, Galicia).

«Mi madre no decía nada». (Elvira, 66, química y geografía/propietaria de hotel, Cataluña). 
«Mi madre no opinaba demasiado...». (Abril, 36, turismo/patronato de turismo, Cataluña).

"Quien llevaba la voz cantante era el padre y la madre siempre ha acatado lo que ha dicho él. Era el padre el que resolvía o el que lo terminaba de estropear...». (Rosalba, 49, trabajo social/profesora de instituto, Cataluña).

\section{LA FORMACIÓN COMO ITINERARIO PERSONAL Y COMO ITINERARIO PROFESIONAL INNOVADOR}

En España y a principios del siglo XX, entre los factores decisivos para la incorporación de las mujeres al mundo educativo, especialmente en la enseñanza media y superior, estuvieron el deseo del cultivo intelectual y la necesidad de contar con un seguro económico para el porvenir (Capel, 1986). En el medio rural, la formación supuso además una estrategia para lograr el ascenso social, para acceder a puestos de trabajo mejores que los de agricultoras y para conseguir, en definitiva, un estatus profesional del que carecerían como mujeres de agricultor. Con la Ley General de Educación de 1970 la escolaridad pasará a ser obligatoria, mixta y gratuita hasta los 14 años. Posteriormente, en los años noventa del siglo pasado se consolidan los cambios experimentados en la educación en las dos décadas precedentes, destacando la extensión de los niveles formativos de las mujeres hacia los estudios superiores (desplazando a los hombres de su posición prioritaria en los estudios universitarios), la universalización de los estudios medios y la expansión de los no obligatorios. Todos estos cambios tuvieron como primera consecuencia que en la actualidad, hombres y mujeres, a excepción de las generaciones anteriores a la Ley del 70, puedan tener una formación básica obligatoria, vivan en el medio rural o en el urbano.

Esto supone, asimismo, un cambio radical en la trayectoria personal y profesional de la población rural, y en particular de la femenina. El contexto por un lado y la influencia de los progenitores, por otro, en particular de las madres, empujan a muchas mujeres a la educación media y superior como vía para alejarse del rural y acceder a un trabajo cualificado y con expectativas de profesionalización. El modelo de agricultura familiar con base patrimonial, muy desigual en cuanto a los roles de género, continúa vinculando a más hombres en las explotaciones en un contexto en que hay muy pocas mujeres. El resultado de la estrategia educativa ha conducido, pues, a un desarraigo femenino del medio rural y a una fuerte masculinización, de forma ininterrumpida hasta hace poco más de una década (García y Sánchez, 2005; Camarero, 2009; García, 2011).

Los avances socioeconómicos de los últimos años han producido un cambio en las formas de pensar de la población joven. Si la educación se utilizó inicialmente para la ascensión social vía huida del campo, ahora esta huida no es tan clara. No es tan directo que las mujeres formadas quieran vivir en la ciudad y puede que este planteamiento sólo se dé cuando no haya una correspondencia entre nivel formativo y posibilidades de trabajo en el medio rural. Las condiciones materiales de vida han hecho más plausible la vida en el medio rural ofreciendo opciones diversas para trabajar (más vinculadas al commuting que a la oferta de trabajos cualificados localizados), y la construcción de una imagen de lo rural más positiva desde lo urbano ha provocado nuevos sentimientos de apego (Díaz, 2005, 2006; Villarino et al., 2013). Las mujeres de nuestro estudio son un ejemplo de esta situación. 
Las tres cuartas partes de las mujeres entrevistadas tienen estudios universitarios e incluso algunas de ellas con nivel de máster o doctorado, que realizaron, en el caso de las catalanas, mayoritariamente en Barcelona (algunas más jóvenes en Tarragona y Girona), y en el caso de las gallegas mayoritariamente en Santiago de Compostela y en menor medida en Coruña y Vigo. Esta etapa formativa fue fundamental en estas mujeres no solo por la adquisición de conocimientos sino por vivir en el ambiente urbano y por asumir con total naturalidad la necesidad de desplazarse y de vivir en otros lugares para formarse.

«Has de hacer alguna práctica en alguna bodega que no sea la tuya y, si la hacía en época de vendimia europea, dejaba tirados a los de casa. Entonces, hice aquí la vendimia y, después de Navidad, fui al hemisferio sur». (Mariona, 29, ingeniería técnica agrícola y enología/agricultora, Cataluña).

La vida en la periferia ha sido un obstáculo que, ciertamente, ha condicionado su trayectoria:

« ¿Claro que condiciona! (...). Tal vez si estuviera en un sitio más cercano a una universidad, estaría ya estudiando aunque fuera a paso lento». (Paz, 41, bachillerato/administrativa en empresa privada, Galicia).

«Por ejemplo, para formarme, muchas veces tengo que desplazarme a Madrid o a Bilbao, y eso tiene un coste muy grande». (Asunción, 43, química/consultora medioambiental, Galicia).

Por ello el espacio virtual ha sido fundamental en su formación y profesionalización:

Cuando empecé a trabajar como traductora el mundo había cambiado completamente y encontré un grupo muy bueno de formación a través de internet. Eso me abrió muchas puertas. Es una asociación sin ánimo de lucro que ofrece formación y apoyo para traducción e intérpretes. Con ellos aprendí mucho y fui haciendo muchas cosas». (Aileen, 43, traducción e interpretación/traductora, Cataluña).

«Luego me decidí a tener una titulación oficial e hice un posgrado de Community Management on-line por la Universidad de Barcelona y por la Business School, y lo acabé en diciembre». (Rosa, 32, filología gallega/gestora de comunicación on-line, Galicia).

Con todo, y pese a los estereotipos de género y también a sus expectativas iniciales, las mujeres han desarrollado profesiones que han supuesto todo un desafío al rol de la mujer en la esfera del trabajo productivo:

«Hice ingeniería técnica de telecomunicaciones e ingeniería superior de electrónica. Yo llegué un poco conducida por mi hermana que estudió lo mismo. Yo veía lo que hacía y me gustaba, y luego una profe que tenía en Girona nos dio una 
asignatura que me gustó mucho y entonces me decidí. [Qué querías ser de mayor cuando eras pequeña?] Profesora». (Raquel, 27, ingeniera telecomunicaciones y electrónica/técnica vivero empresas, Cataluña).

«Lo primero que recuerdo es que quería hacer helados. Mi madre siempre me decía que yo sería profesora, pero en el colegio tampoco lo tenía claro: me gustaban las ciencias y los idiomas. Cuando tuve que ir a la universidad, mi primera opción era traducción y la segunda, bioquímica». (Aileen, 43, traducción e interpretación/traductora, Cataluña).

$\mathrm{Y}$ algunas de estas profesiones se relacionan muy directamente con el medio en que se han desenvuelto, ellas y sus familias:

«Yo siempre he estado muy comprometida. En mi casa había vacas, y cuando tu vives del campo, no es que tu padre y tu madre sean agricultores, sino que es toda una vida. Toda la familia vive del campo porque tiene las responsabilidades de lo que le toca. De alguna manera te afecta porque, cuando creces, tus responsabilidades familiares y de gestión de la explotación crecen también. [...] Eso hace que aprendas a querer y a valorar todo este entorno y la vida que tienes. Lo que tenía claro es que no podía ganarme la vida siendo agricultora». (Diana, 43, doctora en ingeniería agrícola; máster en ciencias políticas/investigadora en alimentos, Cataluña).

«Mi padre tenía una fábrica y necesitaba un químico y por eso estudié química, para ayudar a mi padre en la fábrica. De pequeña era dócil; ha sido de mayor cuando he ido definiendo mis ideas». (Elvira, 66, química y geógrafa/propietaria de hotel, Cataluña).

«[Sus estudios son] Ingeniería técnica agrícola; fui a Barcelona porque había hortofruticultura y en Girona hay agropecuaria. Después hice tres años más que son los de enología y, después de los tres, necesitas el superior de enología, que son dos años y los hice en Tarragona». (Mariona, 29, ingeniería técnica agrícola y enología/agricultora, Cataluña).

\section{IV.1. Autoconcepto y capacidad para la innovación}

El concepto que tienen las mujeres de sí mismas, mediatizado por el entorno familiar y social, como se ha expuesto, explica en buena parte su capacidad para emprender e innovar. Las mujeres se ven a sí mismas como curiosas, dispuestas a saber más, a comenzar nuevos proyectos, a tener la mente abierta y a ser creativas. Esa actitud les permite ver las posibilidades que tiene el medio rural para poder vivir en él y las conduce a explorar lo que se hace en otros lugares y lo que se puede adaptar a los suyos. Además, las mujeres también se definen como emprendedoras, con ganas de hacer cosas, arriesgadas, y dispuestas a hacer lo que sea para salir adelante. Finalmente, se autodefinen también como 
trabajadoras, muy trabajadoras, sin miedo al trabajo y positivas. Estas características que ven en ellas mismas les otorgan el empuje y capacidad necesarios para liderar proyectos y hacer frente a nuevos retos.

«Lo que tienes que hacer es creer en tu proyecto, aunque no hagas estudios de nada; decir voy a por esto y seguir... Porque si yo hubiera hecho estudios de mercado, no estaría aquí porque todos hubieran salido negativos. (...). Hay que ser tenaz y caminar sin mirar hacia atrás; siempre hacia adelante». (Mar, 41, ciencias empresariales/propietaria ganadería ecológica y galletería, Galicia).

«Un hombre no hubiese hecho esto. No lo hubiese llevado de esta forma. En 25 años hacer un patrimonio de 5 millones de euros, no lo hace un hombre; es imposible. [¿Por qué?] Por el esfuerzo que representa hacer todo esto: la visión, el tener siempre el trabajo en la cabeza... Un hombre no tiene esta capacidad». (Coloma, 61, secretariado dirección/gerente centro de educación especial, Cataluña).

En esta imagen de sí mismas, las trayectorias de las madres son importantes:

«En casa de mi madre eran agricultores y había un hijo y una hija; el hijo fue el hereu. Y es algo que no terminas de aceptar. Ahora el hijo, mi tío, pasa las tierras a sus hijas y si no las trabajan las perderán. Y a mí no me tocó nada! Es que estas cosas todavía están muy enraizadas, eh!». (Carla, 41, historia; máster en archivística; máster en museología y gestión de patrimonio/subdelegada patrimonio del obispado, Cataluña).

«Un poco de todo... Mi madre era empresaria. Cuando éramos pequeños tenían un bar-restaurant y, después, mi madre abrió una tienda y un pequeño café». (Aileen, 43, traducción e interpretación/traductora, Cataluña).

Y la formación continúa siendo su motor, punto de partida y fuente de seguridad:

«...el máster me ayudó mucho porque fue un MBA Executive, fue en el año 2003, cuando ya el proyecto estaba en marcha; busqué esta formación específica y me ayudó mucho porque me abrió unas miras muy grandes: de lo que es el trabajo en equipo, de lo que es una organización aunque sea de una PYME, de lo que debe exigírsele a un gerente o a un director... Esa [formación] fue la más específica». (Natalia, 43, ciencias económicas y empresariales; máster MBA/propietaria pazo, Galicia).

«Hacía todo lo que salía: por la asociación de empresarios, Turgalicia... todo lo que saliera y no costara mucho dinero. ¡Fui a todo! (...) [ahora] estoy haciendo un curso nuevo de dirección de cocina de FP. Es de ciclo superior y es todo un curso. Voy por la mañana, voy por la tarde...» (Mariña, 43, ciencies económicas/ propietaria turismo rural, Galicia). 


\section{IV.2. El impacto en el entorno}

Las mujeres utilizan su posición como emprendedoras para cambiar sus vidas y las de los otros, y en ese proceso cambian los lugares en los que viven (Hanson, 2009). En unos casos creando puestos de trabajo, cuantitativamente poco relevantes pero muy importantes en unos territorios con poca población y con un grado muy elevado de pluriactividad familiar.

«En el aserradero trabajan 9 personas. Son 9 personas que viven en el medio rural, que llevan su sueldo a casa y lo necesitan para sobrevivir». (Carolina, 43, ciencias empresariales/técnica aserradero, Galicia).

«Estoy yo, está Mercedes -mi socia- y está Chus. Esperamos poder contratar pronto al hijo de Mercedes. Nos hemos montado nuestra historia: si el hijo de Mercedes está en paro, a ver si lo podemos contratar... Son este tipo de cosas». (Mar, 41, ciencias empresariales/propietaria ganadería ecológica y galletería, Galicia).

«La marca Pazo de Vao apuesta por la calidad, el trabajo artesanal, la agricultura ecológica, la reforestación autóctona y la creación de empleo local y de empleo para mujeres, en un sector deprimido como es el agrícola. (...) En las 37 ha en producción que conforman Pazo de Vao trabajan más de 25 personas». (Natalia, 43, ciencias económicas y empresariales; máster MBA/propietaria pazo, Galicia).

El hecho de ser empresaria o de trabajar en una actividad muy masculinizada es una transgresión de los estereotipos de género y por lo tanto es un factor de cambio porque actúa como efecto demostración.

«La primera vez que fui a una subasta de madera, hace cerca de 20 años, las únicas mujeres que había en la sala éramos la acomodadora y yo.(...). Llegan a un trabajo y se encuentran a una mujer cuando lo que esperan encontrar es un hombre. Todo lo que está relacionado con la madera son trabajos típicamente masculinos... Ahora, por suerte, la mayoría de granjas ya las llevan las mujeres y no los hombres; ellas siempre estaban ahí, pero nunca estaban... Ahora, por suerte, firman y son las dueñas». (Carolina, 43, ciencias empresariales/técnica aserradero, Galicia).

Y ese efecto demostración desde las mujeres empresarias se traslada a otras mujeres que a partir de su trabajo ganan autoridad dentro y fuera del ámbito familiar, aumentan su movilidad espacial, mejoran su situación económica, y lo que es más importante, aumentan su autoestima, su empoderamiento. Es un proceso que desemboca en el cambio en las relaciones de género.

«Fue increíble [referido a los cambios experimentados por una empleada]; es como otra persona. Además, fue una situación de partida difícil porque era una persona 
que nunca había trabajado fuera de casa y, de repente, cogió un coche cuando llevaba sin conducir veinte años. Pudo demostrar que tiene un trabajo y que puede hacerlo; que le costó mucho al principio, pero que lo hizo: un día, otro día, otro día. (...). Ella cobra más que su marido. Mira hacia atrás y puede decir: lo conseguí. Pude sacarme el carnet, pude sacar adelante un trabajo que me parecía imposible y, por encima de todo, mi marido comparte las tareas. (...) [Y eso le ocurre a más mujeres] A mucha gente que trabaja en el balneario le pasa lo mismo... Muchas mujeres de una determinada edad; esa edad en que no eres ni joven ni mayor». (Mariña, 43, ciencias económicas/propietaria turismo rural, Galicia).

«Cuando voy a vender vino a algún restaurante o a cualquier otro sitio, me siento mejor acogida que si fuera mi marido. [Por qué?] No lo sé... Supongo que porque ven a una mujer que les habla del tiempo de crianza, de la fermentación... Una mujer que está en un mundo que es mucho más de hombres. [¿Mayoritariamente tratas con hombres?] Sí; la mayoría son hombres, aunque cada vez me encuentro a más mujeres, iy muy jóvenes;» (Meritxell, 41, bellas artes/propietaria de bodega, Cataluña).

Y un importante e innovador factor de cambio local es la generación de redes de intercambio entre todo el empresariado, intercambio en el que tienen mucho que ver estas empresarias a las que, por supuesto interesa su empresa, pero que también ven en estas redes de proximidad la forma de crear un territorio inteligente con proyección exterior.

«Como aquí nos quedamos unos cuantos, en Ferrera también pasó. Lo bueno que hubo aquí fue que los que nos quedamos creamos lo de los artesanos. Eso hizo que viniera la gente a conocernos. Ahora, desde las entidades-Consejos Comarcales, Ayuntamientos...- nos traen gente: "Id a Ossera, que es el pueblo de los artesanos». Se dice: «En cada casa hay un artesano». (Neus, 56, maestra/artesana neorural, Cataluña).

«Considero que está habiendo muchos ganaderos que se quieren meter al tema ecológico; entonces vienen, preguntan... En nuestra granja, todo lo que hacemos es ecológico (producción vegetal, animal y nuestra industria alimentaria) y se toma como ejemplo de que es factible. Vienen a informarse y les ayudo. Además, en la comarca somos un montón de gente activa y nos ayudamos entre todos. Si, por ejemplo, viene un programa aquí, yo les digo hablad con estos, nos damos publicidad entre todos, utilizamos las mismas tiendas para introducir los productos de nuestros amigos... Yo, en este sentido, sí que considero que es un beneficio común». (Mar, 41, ciencias empresariales/propietaria ganadería ecológica y galletería, Galicia).

En las políticas públicas respecto al medio rural, uno de los objetivos reiteradamente señalados es la mejora de la calidad de vida para que no haya diferencias entre la población en el acceso a los servicios según el lugar de residencia (Directrices Estratégicas Comunita- 
rias de Desarrollo Rural para el período 2007-2013, LEY 45/2007, de 13 de diciembre, para el desarrollo sostenible del medio rural). Es más frecuente que sean las mujeres las que identifiquen las necesidades cotidianas de la población local porque suelen ser ellas las que han de resolverlas en el día a día. Por ello no es casual que entre las mujeres entrevistadas haya algunas cuya iniciativa laboral esté en esa línea y lo que tratan es de cubrir una demanda de la población y así mejorar la calidad de vida en el lugar.

Con el transporte escolar en una zona de montaña:

«(...) montamos el transporte escolar. El primer año los llevábamos en coche y nos lo combinábamos para llevarlos; ahora ibas tú, ahora iba otro... Entonces montamos el transporte escolar y como Alfonso tenía el carnet de autocar hacía de chófer; y como hacía falta que fuese un acompañante, yo hacía de acompañante. Eso lo hicimos durante todos los años de la enseñanza de nuestros hijos, que me parece que fueron 13. [¿Había niños?] Sí. Los nuestros fueron los primeros, después se unieron los dos de Suzette, en Padrinàs había uno o dos niños más, y ya recogíamos a todos los de la Vall. Llegamos a llevar, en los años fuertes, 15 ó 16 niños». (Neus, 56, maestra/artesana neorural, Cataluña).

Con un centro de educación especial y taller ocupacional:

« ¿Empezamos en el 86! En el año 98 ya teníamos 35 personas entre el centro ocupacional y el centro especial de trabajo. (...) Siempre hemos trabajado para la máxima integración. Para mí eso era un atractivo para la comarca... (...) Hay muchos niveles. Hay el que ha de hacer un proceso de formación para poder estar en un centro especial de trabajo, que es un lugar en el que tienen una nómina y una seguridad social. (...) Ahora tenemos el taller ocupacional... Comienzan por la terapia ocupacional y después los pasamos al servicio ocupacional de inserción». (Coloma, 61, secretariado dirección/gerente centro de educación especial, Cataluña).

O con un centro de día para personas mayores:

«Un centro de día estaba más visto a los cuidados, entonces yo veía que hacía falta algo más de dinamismo. Por ejemplo, que se hiciera más gimnasia, una sala de rehabilitación, ya que la hay pues que la utilicen, no que solamente esté ahí por decoración, y bueno, una merienda que no siempre sea lo mismo. Por ejemplo cuando toca descafeinado les hacemos el taller de cocina para que ellos se hagan un bizcocho y se lo coman luego con el descafeinado. Hacemos varios talleres de cocina, como hicimos el otro día, fresas con yogur, pues ellos pelaron las fresas, las mezclaron (...) que ellos sigan con su autonomía. Que se suele vender mucho pero no se suele hacer. Son pequeños detalles que les gustan». (Lola, 28, trabajo social; máster en gerontología, dependencias y dirección de centros/directora centro de día, Galicia). 
La identificación de los problemas cotidianos y el pragmatismo que caracteriza a las mujeres para resolverlos hace que gestionen lo público también de manera diferente, muy apegadas al lugar y al beneficio de la comunidad, aún en circunstancias poco favorables.

«Por ejemplo, este sábado hacemos un concierto, pero no tenemos dinero. Entonces, lo que hacemos es que tenemos un local muy chulo y contactamos con músicos locales que tocan de manera gratuita para nosotros. Nosotros tenemos música gratuita, organizamos un bar y todo lo que recaudamos se lo damos a ellos. ;Y al final resulta bastante! Todos ganamos y tenemos una fiesta fantástica. Y nuestros hijos pueden crecer viendo que podemos hacer cosas sin pagar por todo». (Rosanna, 45, filología inglesa/empresaria de turismo rural y alcaldesa, Cataluña).

Y por supuesto la formación les ha permitido crear proyectos profesionales innovadores reinventando las actividades tradicionales. Los conocimientos adquiridos y la formación continuada les permiten seguir innovando de cara al futuro, factor clave para la continuidad y el dinamismo del medio rural.

«Por ejemplo, Marta con su granja; cuando alguien tiene una granja, se dedica a los becerros, a la leche, a la carne y punto. Marta está con lo de las galletas, que es dar otra visión a lo que estás haciendo. Los de las queserías también abrieron un mundo viviendo en el campo y dentro de un sector que apenas se movía. $O$ Chusa y Carmela con las plantas. Abren nuevos caminos con lo que tienen. Se puede hacer lo de siempre o cosas nuevas: vender carne y leche, o las galletas; plantar judias y lechugas, o plantar hierbas medicinales... Son gente que abre caminos nuevos con lo que había ya ahí». (Carolina, 43, ciencias empresariales/ técnica aserradero, Galicia).

«;Yo tengo un reto enorme! Hago vida de agricultora, súper tradicional y, la verdad, es que la vida de agricultora es lo último. Mi reto ahora mismo es que se valore por lo que es. El trabajo que supone, el valor añadido que tiene culturalmente, el hecho de conservar una tradición, que estamos trabajando con variedades autóctonas... A mí me es igual que el mejor vino del mundo sea el de Robert Parker, pero que la gente de aquí de la restauración y de los alrededores lo valoren como una opción más, para mí ése es el reto.(...) Yo tengo una ventaja y es que he estudiado enología». (Mariona, 29, ingeniería técnica agrícola y enología/agricultora, Cataluña).

\section{CONCLUSIONES}

El papel de la formación se ha demostrado imprescindible para abordar tanto el proyecto laboral de estas mujeres como su proyecto de vida en el medio rural. Les ha permitido situarse como modelo de la «mujer rural actual» en el territorio rural del siglo XXI (ruralidad ampliada). 
La evolución de la legislación educativa en España tuvo como resultado que la mayoría de las mujeres objeto de esta investigación (en razón de la edad) accedieran a la educación básica obligatoria. Posteriormente, la influencia de la familia las orientó a seguir estudios medios y superiores, algo contemplado, en la mayoría de casos, como la vía de ascenso social. La influencia de las madres se concreta en el afán que tienen para que sus hijas estudien porque ven en la educación/formación el modo de independizarse, de acceder a una profesión, de no ser mujer de agricultor y, en algunas de ellas, de abandono del medio rural.

Los estereotipos de género están absolutamente interiorizados en estas mujeres tal como demuestran las expectativas profesionales que tenían cuando eran pequeñas. Sin embargo, la etapa formativa, la vida en otros lugares y la influencia de los padres y sobre todo de las madres, va mudando estos estereotipos de tal modo que estas mujeres abordan una formación muy variada alejada en ocasiones de lo tipificado como femenino.

Esta idea se refleja en el concepto que tienen de sí mismas, viéndose capaces de abordar proyectos laborales innovadores, en un medio a veces hostil, y venciendo las barreras de género y el control social, muy intensos en el medio rural. En los rasgos con los que se autodefinen se unen los que singularizaron a la mujer rural tradicional: muy trabajadora, dispuesta a salir adelante, haciendo frente a todo (familia, explotación) y los que singularizan a esta nueva mujer rural: curiosas, dispuestas a saber más, a comenzar nuevos proyectos, a tener la mente abierta y a ser creativas.

La postura que apoya que la educación y la formación fueron para las mujeres rurales un elemento de desarraigo y desapego del medio rural y, en definitiva, de abandono del mismo, ahora se revierte porque precisamente la educación y la formación se convierten en factores indispensables para construir las nuevas identidades rurales femeninas. La obligación de vivir en la ciudad para formarse ha sido fundamental en muchos sentidos: han aprendido y han analizado lo rural con la distancia necesaria para valorar qué necesita y qué pueden aportarle, asumiendo la conexión rural-urbano y la movilidad real y virtual como algo natural. Su capital social las empodera y las convierte en agentes fundamentales para el cambio en el medio rural, un cambio que entienden tiene que protagonizar la misma población.

El freno al despoblamiento y los efectos de sus actividades en términos de productos, recursos, servicios, conocimiento, red de relaciones y empleo son muy importantes para el medio rural, tanto como el efecto demostración que pueden ejercer sus acciones en otras mujeres y generaciones. Las mujeres transgreden los estereotipos de género situándose al frente de iniciativas y negocios, a menudo en sectores masculinizados, y en su trayectoria también contribuyen al cambio de las relaciones de género en otras familias y en la comunidad rural.

A pesar de que el contexto les ha sido favorable, las mujeres han ejercido un desafío importante como mujeres. Y este desafío no hubiese sido posible sin la autoestima y empoderamiento que les ha otorgado la formación en el entorno urbano. Con este capital se han consolidado como profesionales y han superado barreras de género y de clase. Y en el origen de la formación está una motivación personal, unas decisiones y unos acontecimientos que sólo se explican desde unas relaciones de género determinadas en la unidad familiar en la que han crecido. 


\section{AGRADECIMIENTOS}

Nuestro especial agradecimiento a todas las mujeres de las comarcas estudiadas, protagonistas de nuestro estudio, y a las personas entrevistadas como informantes clave. A Mónica Carbó, por su colaboración en el proceso de transcripción y codificación del material empírico.

\section{REFERENCIAS BIBLIOGRÁFICAS}

ALDOMÀ, I. (Dir.) (2009): Atles de la nova ruralitat, Lleida, Fundació Món Rural.

BAYLINA, M. y SALAMAÑA, I. (2006): «El lugar del género en geografía rural», Boletín de la Asociación de Geógrafos Españoles, $\mathrm{n}^{\circ}$ 41, 99-112.

BARBERÁ, E. (2005): Psicología y género. Madrid, Prentice Hall.

BETZ, N.E. y HACKETT, G. (1986): «Applications of self-efficacy theory to understanding career choice behavior», Journal of Social and Clinical Psychology, 4, 279-289.

BOCK, B. (2004): «It still matters where you live: Rural women's employment throughout Europe», en H.Buller y K. Hoggart (eds.), Women in the European countryside, Aldershot, Ashgate, pp. 14-41

BORDIEU, P. (2004): El baile de los solteros, Barcelona, Anagrama (original francés 2002).

CAMARERO, L. (Coord.) (2009): La población rural de España. De los desequilibrios a la sostenibilidad social. Colección Estudios Sociales, $\mathrm{n}^{\circ}$ 27. Barcelona, La Caixa.

CANDELA, C., BARBERÁ, E. y RAMOS, A. (2009): «Perspectiva de género en el desarrollo de carrera:perfil de competencias, logro de metas y dificultades encontradas», Información Psicológica, $\mathrm{n}^{\circ}$ 97, 27-36.

CANOVES, G., VILLARINO, M., PRIESTLEY, G. y BLANCO, A. (2004): «Rural tourism in Spain: an analysis of recent evolution», Geoforum, n 35, 755-769.

CAPEL, R. (1986): El trabajo y la educación de la mujer en España (1900-1930), Madrid, Instituto de la Mujer.

CARBÓ, M., BAYLINA, M. y GARCIA RAMON, M.D. (2014), «Una mirada de género a la geografía rural española. Mujeres, negocios e identidad rural en Cataluña», en H. Castro y M. Arzeno (Coord.), Temas y perspectivas en Geografía Rural, Buenos Aires, Editorial de la Facultad de Filosofía y Letras, Colección Cátedra (en prensa).

CASELlAS, A., TULLA, A., VERA, A. y PALLARÈS-BLANCH, M. (2013): «Gobernanza local y espacio rural: un análisis territorial desde la perspectiva de género», Boletín de la Asociación de Geógrafos Españoles, ${ }^{\circ}$ 62, 379-402.

DEAUX, K. y LEWIS, L. (1983): «Components of gender stereotypes», Psychological Documents, $\mathrm{n}^{\circ} 13,25$.

DÍAZ, C. (2005): «Aproximaciones al arraigo y al desarraigo femenino en el medio rural: mujeres jóvenes en busca de una nueva identidad rural», Papers $\mathrm{n}^{\circ}$ 75, 63-84.

DÍAZ, C. y DÁVILA, M. (2006), Familia, trabajo y territorio, Madrid, Ministerio de Agricultura, Pesca y Alimentación.

DRAE (Diccionario de la Real Academia Española) (2001) (22 a edición).

EAGLY, A. H. y STEFFEN (1984): «Gender stereotypes stem from the distribution of women and men into social roles», Journal of Personality and Social Psychology, 46(4), 735-754. 
ECCLES, J.S. (1994): «Understanding women's educational and occupational choices», Psychology of Women Quarterly, 18, 585-609.

ELEJABEITIA, C. y LÓPEZ-SÁEZ, M. (2003): Trayectorias personales y profesionales de mujeres con estudios tradicionalmente masculinos. Madrid: CIDE-Instituto de la Mujer.

GARCÍA, B. (2011): Ruralidad emergente, posibilidades y retos, Madrid, Ministerio de Medio Ambiente, Medio Rural y Marino.

GARCÍA, A. y SANCHEZ, D. (2005): «La población rural en Catalunya: entre el declive y la revitalización», Cuadernos Geográficos, $\mathrm{n}^{\circ} 36,387-407$.

GARCIA RAMON, M.D. y BAYLINA, M. (Eds.) (2000): El nuevo papel de la mujer en el desarrollo rural, Vilassar de Mar, Oikos-Tau.

GARCÍA SANZ, B. (2004): La mujer rural ante el reto de la modernización de la sociedad rural, Madrid, Ministerio de Trabajo y Asuntos Sociales, Instituto de la Mujer.

HANSON, S. (2009): «Changing places trough women’s entrepreneurship», Economic Geography, vol. 85, 3, 245-267.

HERNÁNDEZ FRANCO, V. (2001): Análisis causal de los intereses profesionales en los estudiantes de secundaria. Madrid: Tesis Doctoral. Universidad Complutense de Madrid.

HOGGART, K. y PANIAGUA, A. (2002): «The restructuring of rural Spain?», Journal of Rural Studies, $\mathrm{n}^{\mathrm{O}} 17,63-80$.

HOLLINGER, C.L. (1983): «Self-perception and the career aspirations of mathematically talented female adolescents», Journal of Vocational Behavior, 22, 49-62.

HOPKINS, J. (2010): Young people, place and identity, London, Routledge.

HYDE, J. S. et al. (1990): «Gender comparisons of mathematics attitudes and affect: A metaanalysis», Psychology of Women Quarterly, 14, 299-324.

JOZEFOWICZ, D. M., BARBER, B.L. y ECCLES, J. S. (1993): Adolescent work related values and beliefs: Gender differences and relation to occupational aspirations. Paper presented at Biennial Meeting of the Society for Research on Child Development. New Orleans, LA.

MARTIN,CC., WOOD CH. y LITLTE,J.K. (1990): «The development of gender stereotype components» Child Development, 61 (6), 891-904.

MINISTERIO DE MEDIO AMBIENTE Y MEDIO RURAL Y MARINO (2011): Diagnóstico de la Igualdad de Género en el Medio Rural, Madrid.

MORRIS, C. y LITTLE, J. (2005), «Rural work: An overview of women's experiences», Critical studies in rural gender issues, Aldershot, Ashgate, pp. 9-26.

MOSTEIRO GARCÍA, M.J. (2010): «Los estereotipos de género y su transmisión a través del proceso de socialización», en Radl Phillip, R. (Ed.): Investigaciones actuales de la mujer y del género, Santiago de Compostela, Servicio de Publicaciones e Interambio Científico de la Universidad de Santiago de Compostela, pp. 239-252.

NASH, M. (1995): «Identitat cultural de gènere, discurs de la domesticitat i definició del treball de las dones a 1'Espanya del segle XIX», Documents d'Anàlisi Geogràfica, n 26, 135-146.

NIETO, C. (2004): «Iniciativas empresariales y nuevas oportunidades de empleo en el desarrollo rural. Planteamientos teóricos y casos prácticos en Málaga, Cuadernos Geográfi$\cos , \mathrm{n}^{\circ} 35: 11-27$.

PALLARÈS BARBERÀ, M., TULLA, A., y PALLARÈS BLANCH, M. (2004): Capital social i treball de les dones als Pirineus. El cas de l'Alt Urgell, Barcelona, Institut Català de les Dones. 
PRADOS, M.J. (2000): Situación socioeconómica de las mujeres rurales en España, Sevilla, Junta de Andalucía, Consejería de Agricultura y Pesca.

SAINZ, M.; LÓPEZ-SAÉZ, M.; LISBONA, A. (2004): «Expectativas de rol profesional de mujeres estudiantes de carreras típicamente femeninas o masculinas», Acción Psicológica, 3, 2, 111-123.

SALANOVA, M., HONTANGAS, P. M. Y PEIRÓ, J. M. (1996): «Motivación laboral» en J.M. Peiró y F. Prieto: Tratado de Psicología del Trabajo. Volumen I: la actividad laboral en su contexto, Madrid, Síntesis, pp. 215-249.

SAMPEDRO, R. (2009), «Escuchando la voz de las mujeres», Agricultura familiar en España 2009, Anuario de la Fundación de Estudios Rurales, Unión de Pequeños Agricultores y Ganaderos, pp. 92-102.

SHAFFER, D. (2000): Desarrollo social y de la personalidad. Madrid, Thomson.

SWIM, J. K. (1994): «Perceived versus meta-analytic effect sizes: An assesment of the accuracy of gender stereotypes», Journal of Personality and Social Psychology, 66(1), 21-36.

TULLA, A., CASELlAS, A., PALLARÈS-BLANCH, M. y VERA, A. (2012): «Las relaciones de género en las políticas locales y en el desarrollo económico del Pirineo Catalán», Revista Latino-americana de Geografia e Gênero, Ponta Grossa, 3 (2), 76-86.

VALENTINE, G. (2004): Public space and the culture of childhood, Aldershot, Ashgate.

VALENTINE, G. Y MCKENDRICK, J. (1997): «Children's outdoor play: exploring parental concerns about children's safety and the changing nature of childhood», Geoforum, 28 (2), 219-235.

VAN DER PLOEG et al. (2000), «Rural development: from practives and policies to theory», Sociologia Ruralis, 40 (4): 391-408

VILLARINO, M., BAYLINA, M., GARCIA RAMON, M.D., PORTO, A. y SALAMAÑA, I. (2013): «Pertenencia al lugar y nueva ruralidad. Mujeres profesionales estrechan vínculos con lo rural» en Actas del XXIII Congreso de Geógrafos Españoles, Palma de Mallorca, pp. 719-729.

WILLIAMS, J. E. y BEST, D.L. (1990): Measuring sex stereotypes:A multination study. Newbury Park Sage.

WHATMORE, S. (1990): «Theories and practices for rural sociology in a new Europe», Sociología Ruralis, no 30 (3-4), 251-259.

WOODS, M. (2011): Rural, London, Routledge. 